\title{
Prontuário eletrônico do cidadão: aceitação e facilidade de uso pelos cirurgiões-dentistas da atenção básica
}

Electronic records of citizens: acceptance and ease of use by dentists of basic attention service

Historia clínica electrónica del individuo: aceptación y facilidad de uso por dentistas del servicio de atención primaria Danilo Vieira BARBOSA ${ }^{1}$

Waleska Fernanda Souto NÓBREGA²

Gustavo Correia Basto da SILVA2

Osires de Medeiros MELO NETO ${ }^{1}$

Faldryene de Souza Queiroz FEITOSA ${ }^{1}$

${ }^{1}$ Universidade Federal de Campina Grande - UFCG

${ }^{2}$ Universidade Estadual da Paraíba - UEPB

\section{Resumo}

Objetivo: Avaliar a aceitação e facilidade de uso do Prontuário Eletrônico do Cidadão pelos Cirurgiões Dentistas da Atenção Básica. Metodologia: O estudo foi do tipo transversal, descritivo e analítico, tendo como população alvo os Cirurgiões Dentistas que atuam nas Unidades Básicas de Saúde de uma cidade do interior do Nordeste. Para coleta de dados foi utilizado um formulário estruturado, com questões objetivas e autoadministradas. Os dados obtidos através dos questionários respondidos foram digitados e tabulados no software SPSS 22.0 e analisados com base em estatísticas descritivas e análise inferencial através do teste qui-quadrado admitindo-se significância de $5 \%(\mathrm{p} \leq 0,05)$. Resultados: A maioria dos entrevistados era do sexo feminino (63\%), com idade entre 31-50 anos $(44,4 \%)$, formados entre $0-5$ anos $(51,9)$, com menos de 5 anos de trabalho nas unidades $(63 \%)$. A maioria (63\%) julgou o prontuário eletrônico fácil de usar. Houve diferença estatisticamente relevante na questão sobre dificuldade do uso do PEC e o tempo de atuação na Unidade, entre o esforço utilizado para adaptação ao PEC e o tempo de formação e ainda entre achar o PEC de difícil uso em geral com a idade do participante. Conclusão: O PEC foi, em geral, bem aceito e amplamente utilizado pelos profissionais da saúde bucal, havendo algumas lacunas para melhoria do uso como o tempo de treinamento.

Descritores: Registros Eletrônicos de Saúde; Atenção Primária à Saúde; Sistemas de Informação.

\section{Abstract}

Aim: To evaluate the acceptance and ease of use of the Citizen's Electronic Health Record by Primary Care Dentist Surgeons. Methodology: The study was cross-sectional, descriptive and analytical, having as target population the Dental Surgeons who work in the Basic Health Units of a city in the northeast of the Northeast. For data collection, a structured form was used, with objective and self-administered questions. The data obtained through the answered questionnaires were typed and tabulated in the SPSS 22.0 software and analyzed based on descriptive statistics and inferential analysis using the chi-square test, assuming a significance of 5\% ( $\leq 0.05$ ). Results: Most respondents were female (63\%), aged between $31-50$ years $(44.4 \%)$, graduated between $0-5$ years (51.9), with less than 5 years of work in the units. $(63 \%)$. Most (63\%) found the electronic medical record easy to use. There was a statistically significant difference in the question of difficulty in using the PEC and the time worked in the unit, between the effort used to adapt to the PEC and the time of training and between finding the PEC difficult to use in general with the participant's age. Conclusion: PEC was generally well accepted and widely used by oral health professionals, with some gaps to improve use such as training time.

Descriptors: Electronic Health Records; Primary Health Care; Information Systems.

\section{Resumen}

Objetivo: Evaluar la aceptación y la facilidad de uso de la Historia clínica electrónica del ciudadano por parte de los cirujanos dentistas de atención primaria. Metodología: El estudio fue transversal, descriptivo y analítico, teniendo como población objetivo a los cirujanos dentales que trabajan en las unidades básicas de salud de una ciudad en el noreste del noreste. Para la recolección de datos, se utilizó un formulario estructurado, con preguntas objetivas y autoadministradas. Los datos obtenidos a través de los cuestionarios respondidos se tipificaron y tabularon en el software SPSS 22.0 y se analizaron en base a estadísticas descriptivas y análisis inferencial utilizando la prueba de chicuadrado, asumiendo una significancia del $5 \%(\mathrm{p} \leq 0.05)$. Resultados: la mayoría de los encuestados eran mujeres $(63 \%)$, con edades comprendidas entre 31-50 años (44.4\%), graduados entre 0-5 años (51.9), con menos de 5 años de trabajo en las unidades. (63\%). La mayoría (63\%) encontró el registro médico electrónico fácil de usar. Hubo una diferencia estadísticamente significativa en la cuestión de la dificultad para usar el PEC y el tiempo trabajado en la unidad, entre el esfuerzo utilizado para adaptarse al PEC y el tiempo de entrenamiento y entre encontrar el PEC difícil de usar en general con la edad del participante. Conclusión: PEC fue generalmente bien aceptado y ampliamente utilizado por profesionales de la salud oral, con algunas brechas para mejorar el uso, como el tiempo de entrenamiento. Palabras clave: Registros electrónicos de salud, atención primaria de salud, sistemas de información.

Descriptores: Registros Electrónicos de Salud; Atención Primaria de Salud; Sistemas de Información.

\section{INTRODUÇÃO}

Dentre as responsabilidades cotidianas dos profissionais da saúde ligados à área assistencial está o registro das informações da saúde e doença dos pacientes. Tal registro é realizado via documento, prontuário médico ou registro médico, que agrupa informações gerais sobre $\mathrm{o}$ paciente. Com $\mathrm{o}$ surgimento dos meios informatizados, foi possível a criação do Prontuário Eletrônico do Paciente, visando substituir sua versão impressa, almejando aprimoramento no armazenamento de informações e, assim, melhorar os serviços assistenciais de saúde ${ }^{1}$.

$\mathrm{O}$ prontuário eletrônico do cidadão (PEC) utiliza metodologias informacionais e tecnológicas a fim de buscar uma maior resolubilidade dos problemas de saúde por meio dos conhecimentos da ciência da informação e de seus profissionais ${ }^{2}$ e tem sido um dos temas mais abordados em pesquisa e desenvolvimento quando se fala em informática em saúde $e^{3,4}$. Outro benefício do PEC é servir como documento de referência para o processo de comunicação intra e extra equipe de saúde ${ }^{5}$.

O prontuário odontológico, por sua vez, contém informações padronizadas e ordenadas sobre a assistência odontológica e teve seu modelo proposto pelo Conselho Federal de Odontologia (CFO), dividindo-o em documentos fundamentais e suplementares. Esse instrumento fornece informações sobre o paciente avaliado através dos dados contidos 
em seu conjunto de documentos, com finalidade da elaboração de diagnóstico, planejamento, execução e acompanhamento ${ }^{6}$.

O prontuário eletrônico odontológico é considerado um avanço significativo para a comunidade, pois os que utilizam das clínicas como locais de aprendizagem terão, por meio dele, uma fonte mais confiável de informações referentes aos pacientes atendidos ${ }^{7}$.

No final do ano de 2016, o Ministério da Saúde determinou que os municípios brasileiros deveriam adotar o PEC em todos os serviços de Atenção Básica ${ }^{8}$. O PEC do sistema $e$-SUS é um software criado pelo Departamento de Informática do SUS (DATASUS), onde todas as informações clínicas e administrativas do paciente ficam armazenadas, no contexto da Unidade Básica de Saúde (UBS), tendo como principal objetivo informatizar o fluxo de atendimento do cidadão realizado pelos profissionais de saúde ${ }^{9}$.

Essa nova tecnologia tem sido um dos temas mais abordados em pesquisa e desenvolvimento quando se fala em informática em saúde. Porém, atualmente, no Brasil e mais especificadamente na Odontologia, ainda são poucos os estudos publicados quanto a essa temática. O Ministério da Saúde estipulou que as UBSs adotassem o novo sistema, caso contrário, as prefeituras deixariam de receber repasses financeiros para serviços da Atenção Básica $^{10}$.

A destarte, o objetivo geral dessa pesquisa foi avaliar a aceitação e facilidade de uso do Prontuário Eletrônico do Cidadão pelos Cirurgiões Dentistas da Atenção Básica.

MATERIAL E MÉTODO

O presente trabalho foi um estudo observacional, transversal, descritivo e analítico, desenvolvido por meio da aplicação de questionários específicos e objetivos, para a avaliação da aceitação e facilidade de uso pelos CDs frente à implantação do PEC. O estudo foi desenvolvido na cidade de Patos, estado da Paraíba, com o universo composto por Cirurgiões Dentistas de todas as 41 UBSs da cidade, registrados no Conselho Regional de Odontologia do estado e que se encontravam em suas respectivas unidades de saúde.

Foram incluídos na pesquisa todos os CDs que trabalham nas UBSs e excluídos os que se negaram a assinar o Termo de Consentimento Livre e Esclarecido (TCLE), não responderam o questionário em tempo hábil ou entraram no serviço após o início da pesquisa.

Para obtenção dos dados relativos ao perfil, avaliação do conhecimento de informática e à implantação do processo do prontuário eletrônico foi utilizado o questionário proposto por Majewski ${ }^{11}$.

Com o intuito de descrever e analisar as variáveis que influenciam a percepção da utilidade e facilidade de uso do PEC sob o ponto de vista dos Cirurgiões-Dentistas da cidade foi utilizado o questionário proposto por Davis $\mathrm{Jr}^{12}{ }^{12}$ e utilizado por Muylder et al. ${ }^{13}$ (Quadro 1). As questões foram adaptadas à proposta desta pesquisa e foram aplicadas utilizando a escala do tipo Likert.

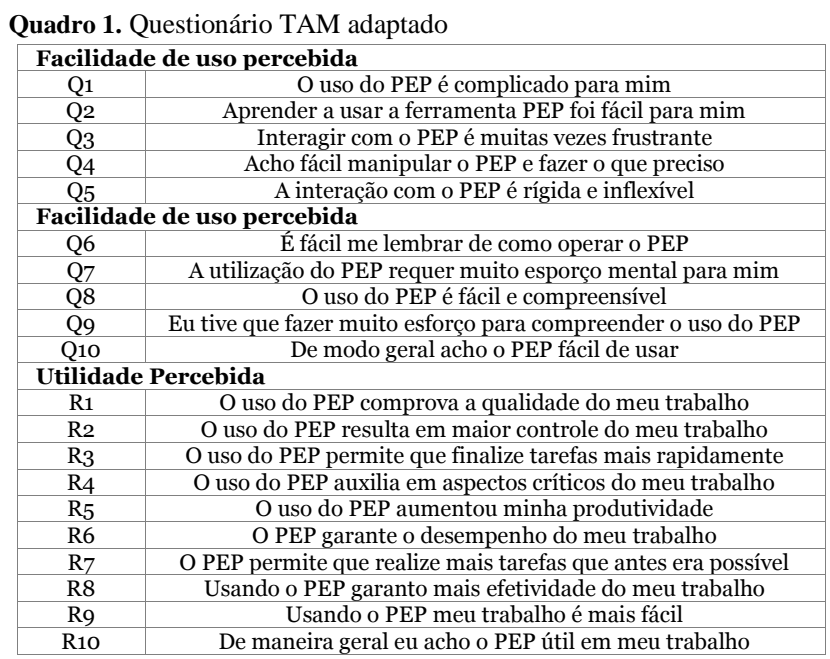

Os resultados foram analisados através do software SPSS 20.0, primariamente aplicando-se a estatística descritiva, posteriormente realizada a análise inferencial dos dados através da aplicação do teste não paramétrico de associação de qui-quadrado $\left(x^{2}\right)$, admitindo-se o nível de significância de 5\% $(p \leq 0,05)$. Os resultados foram expressos em números absolutos e percentuais, apresentados através de Gráficos e Tabelas.

\section{RESULTADOS E DISCUSSÃO}

\section{- Caracterização da amostra}

No presente estudo, conforme se observa na Tabela 1, houve predominância de participantes do sexo feminino com $63 \%(n=17)$ e faixa etária entre 31 e 50 anos com $44 \%(n=12)$.. Com relação ao tempo de formação, 51,9\% (n=14) tem menos de 5 anos de conclusão de curso, enquanto $63 \%(n=17)$ tem menos de 5 anos de atuação na atual Unidade de Saúde. Em se tratando de utilização do computador, $85,2 \%$ dos entrevistados $(n=23)$ relataram o uso fora da Unidade. Acerca da informatização do serviço de saúde, $51,9 \%$ dos participantes do estudo $(n=14)$ acreditam que a saúde opera com um bom nível de informatização, $100 \% \quad(n=27)$ concordam que o investimento em informática melhorou o serviço, $77,8 \%(\mathrm{n}=21)$ sentem- se satisfeitos com o sistema implantado e 96,3\% (n=26) afirmaram que houve diferencial após a implantação do PEC (Tabela 1).

- Conhecimento de Informática e Implantação do PEC

A Tabela 2 mostra que $55,6 \%$ dos entrevistados $(n=15)$ adquiriram conhecimento em informática através de cursos por iniciativa pessoal. Dos participantes 55,6\% $(\mathrm{n}=15)$ relataram que só foram informados do treinamento para uso do PEC 
no dia em que ocorreu. Quanto à realização do treinamento para o uso do PEC, 74,1\% (n=20) afirmaram que o treinamento ocorreu durante $\mathrm{o}$ horário de trabalho com uma empresa. Sobre a carga horária total de treinamento, 66,7\% $\quad(n=18)$ consideraram insuficiente.

Tabela 1. Perfil dos cirurgiões-dentistas e opinião sobre o uso de informática na saúde. Patos, 2018

\begin{tabular}{|c|c|c|}
\hline VARIÁVEIS & $\mathrm{n}$ & $\%$ \\
\hline \multicolumn{3}{|l|}{ Sexo } \\
\hline Masculino & 10 & 37,0 \\
\hline Feminino & 17 & 63,0 \\
\hline \multicolumn{3}{|l|}{ Idade } \\
\hline Ate 30 & 11 & 40,8 \\
\hline 31 a 50 & 12 & 44,4 \\
\hline Maior de 50 & 4 & 14,8 \\
\hline \multicolumn{3}{|c|}{ Tempo de formado } \\
\hline o a 5 anos & 14 & 51,9 \\
\hline $6-10$ anos & 7 & 25,9 \\
\hline Mais de 10 anos & 6 & 22,2 \\
\hline \multicolumn{3}{|c|}{ Tempo de trabalho na UBS } \\
\hline o a 5 anos & 17 & 63,0 \\
\hline $6-10$ anos & 9 & 33,3 \\
\hline Mais de 10 anos & 1 & 3,7 \\
\hline \multicolumn{3}{|c|}{ Uso do computador fora da UBS } \\
\hline Sim & 23 & 85,2 \\
\hline Não & 4 & 14,8 \\
\hline \multicolumn{3}{|c|}{ A saúde opera com bom nível de informatização } \\
\hline Sim & 14 & 51,9 \\
\hline Não & 13 & 48,1 \\
\hline \multicolumn{3}{|c|}{$O$ investimento em informática melhora o serviço } \\
\hline Sim & 27 & 100,0 \\
\hline Não & 0 & o \\
\hline \multicolumn{3}{|c|}{ Satisfação com o sistema implantado } \\
\hline Sim & 21 & 77,8 \\
\hline Não & 6 & 22,2 \\
\hline \multicolumn{3}{|c|}{ O PEP fez diferença no trabalho } \\
\hline Sim & 26 & 96,3 \\
\hline Não & 1 & 3,7 \\
\hline
\end{tabular}

Tabela 2. Conhecimento de informática e processo de implantação do prontuário eletrônico. Patos, 2018

\begin{tabular}{lcc}
\hline VARIÁVEIS & N & $\%$ \\
\hline Como adquiriu conhecimento de informática & & \\
\hline Curso pessoal & 15 & 55,6 \\
Curso oferecido pela gestão municipal & 6 & 22,2 \\
Treinamento direto no sistema oferecido pela gestão & 1 & 3,7 \\
Direto no sistema ajudado por colegas & 0 & 0,0 \\
Outros & 5 & 18,5 \\
\hline Como ficou sabendo do PEP & & \\
\hline Reunião com a gestão municipal & 8 & 29,6 \\
Quando ofereceram o treinamento & 15 & 55,6 \\
Durante a implantação & 2 & 7,4 \\
Após a implantação & 1 & 3,7 \\
Outras & 1 & 3,7 \\
\hline Como foi seu treinamento & & \\
\hline Com uma empresa especializada fora do trabalho & 3 & 11,1 \\
Com uma empresa durante o trabalho & 20 & 74,1 \\
Com a coordenação do setor & 2 & 7,4 \\
Com os colegas & 0 & 0,0 \\
Outros & 2 & 7,4 \\
\hline A carga horária do treinamento foi suficiente & & \\
\hline Sim & 9 & 33,3 \\
Não & 18 & 66,7 \\
\hline
\end{tabular}

\section{- Teste de Facilidade de Uso}

$\mathrm{Na}$ Tabela 3, que discorre quanto ao uso da ferramenta PEC, 77,8\% (n=21) relataram que $o$ mesmo está sempre disponível. Com relação as horas semanais de utilização do PEC, 77,8\% $(n=21)$ disseram usar o sistema entre 20 e 40 horas semanais. Com relação a estabilidade do sistema, 25,9\% ( $\mathrm{n}=7$ ) caracterizaram-na como excelente. Sobre o suporte dado a ferramenta PEC, 51,9\% $(n=14)$ afirmaram ser bom. Com relação ao item de menor qualidade na ferramenta PEC, $37 \%(n=10)$ apontaram as quedas de sistema.
Tabela 3 - Quanto ao uso da ferramenta PEP. Patos, 2018.

\begin{tabular}{lcc}
\hline VARIÁVEIS & N & $\%$ \\
\hline O PEPP está sempre disponível & & \\
\hline Sim & 21 & 77,8 \\
Não & 6 & 22,2 \\
\cline { 2 - 3 } Horas semanais do uso de PEP & 6 & 22,2 \\
\hline Ate 20 h & 21 & 77,8 \\
\hline a 4oh & & \\
\hline Como avalia a estabilidade do PEP & 2 & 7,4 \\
\hline Ruim & 1 & 3,7 \\
Regular & 4 & 14,8 \\
Bom & 13 & 48,1 \\
Muito bom & 7 & 25,9 \\
Excelente & & \\
\hline Como avalia o suporte ao PEP & 3 & 11,1 \\
\hline Ruim & 3 & 11,1 \\
Regular & 14 & 51,9 \\
Bom & 3 & 11,1 \\
Muito bom & 4 & 14,8 \\
Excelente & & \\
\hline Item de menor qualidade do PEP & 7 & 25,9 \\
\hline Desempenho & 10 & 37,0 \\
Erros do sistema & 7 & 25,9 \\
Adaptação do prontuário & 1 & 3,7 \\
Rotatividade de empresas & 2 & 7,4 \\
Atendimento de novos usuários & & \\
\hline
\end{tabular}

$\mathrm{Na}$ tabela 4, que trata sobre a percepção da facilidade do uso de PEC, 55,6\% $(n=15)$ discordaram totalmente que o uso do PEC. Concordaram totalmente com que aprender a usar o PEC foi fácil $44,4 \%(n=12)$. Com relação à frustração de interagir com o PEC, $25,9 \%(n=7)$ discordaram parcialmente e $25,9 \%(n=7)$ discordaram totalmente. Com relação à afirmação que diz que a manipulação do PEC é fácil, $48,1 \% \quad(n=13)$ concordaram totalmente. Dos entrevistados, $37 \%(\mathrm{n}=10)$ discordaram totalmente que a interação com o PEC é rígida e inflexível. Sobre a afirmação que é fácil lembrar como operar o PEC, 29,6\% $(\mathrm{n}=8)$ concordaram totalmente. Com relação à afirmação que o uso do PEC seria fácil e compreensível, $51,9 \% \quad(\mathrm{n}=14) \quad$ concordaram totalmente. $\mathrm{E}$ ainda $44,4 \% \quad(\mathrm{n}=12) \quad$ discordaram totalmente que tiveram de fazer muito esforço para compreender o uso do PEC. Ainda observamos 63\% $(n=17)$ concordaram totalmente que o PEC é fácil de usar.

$\mathrm{Na}$ Tabela 5, que discorre sobre a Percepção de Utilidade do PEC, 25,9\% $\quad(n=7)$ concordam totalmente que o uso do PEC comprova a qualidade do 'trabalho, 44,4\% ( $\mathrm{n}=12)$ concordam totalmente que o uso do PEC resulta em maior controle do trabalho. Por sua vez, 29,6\% (n=8) se mantiveram neutros com relação a afirmação que o uso do PEC finaliza as tarefas mais rápido. Dos participantes entrevistados, 37\% $\quad(n=10)$ se mantiveram neutros diante da afirmativa que diz que o PEC auxilia em aspectos críticos do trabalho, $37 \%$ $(n=10)$ se mantiveram neutros diante da afirmativa que diz que o PEC aumentou a produtividade no trabalho, 33,3\% ( $\mathrm{n}=9)$ discordam totalmente que o PEC garante o bom desempenho do trabalho, 33,3\% $(n=9)$ se mantiveram neutros diante da afirmativa que diz que o PEC permite que as tarefas sejam realizadas de forma mais rápida que antes, 33,3\% (n=9) concordaram parcialmente que o PEC garante maior efetividade do trabalho, 25,9\% $(\mathrm{n}=7)$ concordaram 
totalmente que o uso do PEC tornou o trabalho mais fácil e $55,6 \%(n=15)$ concordaram totalmente que o PEC foi útil ao trabalho.

Tabela 4. Quanto a percepção da facilidade do uso do PEP. Patos, 2018

\begin{tabular}{|c|c|c|}
\hline VARIÁVEIS & $\mathrm{N}$ & $\%$ \\
\hline O uso do PEP é complicado & 2 & 7,4 \\
\hline \multicolumn{3}{|l|}{ Concordo totalmente } \\
\hline Concordo parcialmente & 4 & 14,8 \\
\hline Neutro & 2 & 7,4 \\
\hline Discordo parcialmente & 4 & 14,8 \\
\hline Discordo totalmente & 15 & 55,6 \\
\hline \multicolumn{3}{|l|}{ Aprender foi fácil } \\
\hline Concordo totalmente & 12 & 44,4 \\
\hline Concordo parcialmente & 9 & 33,4 \\
\hline Neutro & 2 & 7,4 \\
\hline Discordo parcialmente & 4 & 14,8 \\
\hline Discordo totalmente & 0 & 0,0 \\
\hline \multicolumn{3}{|l|}{ A interação foi frustrante } \\
\hline Concordo totalmente & 6 & 22,2 \\
\hline Concordo parcialmente & 3 & 11,2 \\
\hline Neutro & 4 & 14,8 \\
\hline Discordo parcialmente & 7 & 25,9 \\
\hline Discordo totalmente & 7 & 25,9 \\
\hline \multicolumn{3}{|l|}{ Fácil manipulação de PEP } \\
\hline Concordo totalmente & 13 & 48,1 \\
\hline Concordo parcialmente & 7 & 25,9 \\
\hline Neutro & 2 & 7,4 \\
\hline Discordo parcialmente & 4 & 14,8 \\
\hline Discordo totalmente & 1 & 3,7 \\
\hline \multicolumn{3}{|l|}{ Interação rígida e inflexível } \\
\hline Concordo totalmente & 2 & 7,4 \\
\hline Concordo parcialmente & 3 & 11,1 \\
\hline Neutro & 4 & 14,8 \\
\hline Discordo parcialmente & 8 & 29,6 \\
\hline Discordo totalmente & 10 & 37,0 \\
\hline \multicolumn{3}{|l|}{ Fácil lembrar como operar } \\
\hline Concordo totalmente & 8 & 29,6 \\
\hline Concordo parcialmente & 4 & 14,8 \\
\hline Neutro & 4 & 14,8 \\
\hline Discordo parcialmente & 4 & 14,8 \\
\hline Discordo totalmente & 7 & 25,9 \\
\hline \multicolumn{3}{|l|}{ Uso fácil e compreensivo } \\
\hline Concordo totalmente & 14 & 51,9 \\
\hline Concordo parcialmente & 3 & 11,1 \\
\hline Neutro & 3 & 11,1 \\
\hline Discordo parcialmente & 7 & 25,9 \\
\hline Discordo totalmente & 5 & 18,5 \\
\hline \multicolumn{3}{|c|}{ Requer muito esforço para compreensão } \\
\hline Concordo totalmente & 5 & 18,5 \\
\hline Concordo parcialmente & 3 & 11,1 \\
\hline Neutro & 3 & 11,1 \\
\hline Discordo parcialmente & 4 & 14,8 \\
\hline Discordo totalmente & 12 & 44,4 \\
\hline \multicolumn{3}{|l|}{ De forma geral o PEP é fácil } \\
\hline Concordo totalmente & 17 & 63,0 \\
\hline Concordo parcialmente & 6 & 22,2 \\
\hline Neutro & 0 & o \\
\hline Discordo parcialmente & 1 & 3,7 \\
\hline Discordo totalmente & 1 & 11,1 \\
\hline
\end{tabular}

Na Tabela 6, observamos a análise referente ao teste qui-quadrado de Pearson $\left(x^{2}\right)$. Na questão que afirmou que era difícil o uso do PEC correlacionado com o tempo de atuação do cirurgião-dentista na unidade, encontramos p valor $=0,036$, bem como na correlação das respostas da afirmativa que dizia que houve muito esforço para aprender a trabalhar com o PEC, com o tempo de formação, onde encontramos o $p$ valor $=0,015$, bem como na correlação entre as respostas da questão que diz que de forma geral acha difícil o uso do PEC com a idade, onde encontramos $\mathrm{p}$ valor $=0,008$.

Não se observou diferença estatisticamente significante entre a percepção da utilidade do uso PEC com nenhuma variável do perfil dos Cirurgiões-Dentistas.
Tabela 5.Quanto a percepção da utilidade do uso do PEP. Patos, 2018

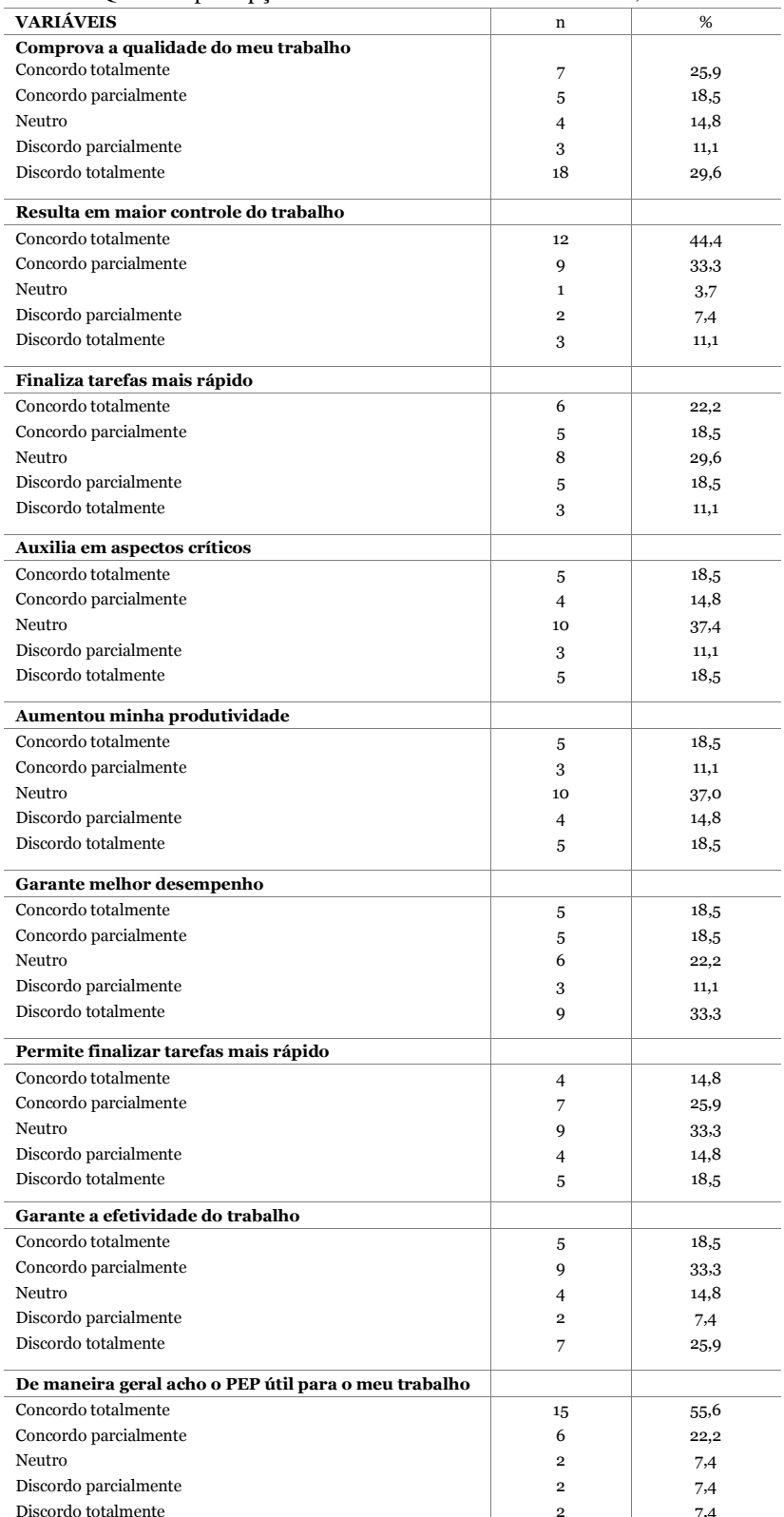

Tabela 6. Relação da percepção da facilidade do uso do PEP com variáveis do perfil dos Cirurgiões-Dentistas. Patos, 2018

\begin{tabular}{|c|c|c|c|c|c|c|}
\hline & & \multirow{2}{*}{$f$} & \multirow{2}{*}{$\%$} & Idade & $\mathrm{TF}$ & $\mathrm{TU}$ \\
\hline & & & & p valor & P valor & $P$ valor \\
\hline O uso d & omplicado & & & & & \\
\hline & Concordo Totalmente & 2 & 7,4 & & & \\
\hline & Concordo parcialmente & 4 & 14,8 & & & \\
\hline Valido & Neutro & 2 & 7,4 & 0,765 & 0,610 & $0,036^{*}$ \\
\hline & Discordo parcialmente & 4 & 14,8 & & & \\
\hline & Discordo totalmente & 15 & 55,6 & & & \\
\hline Tive $\mathrm{m}$ & & & & & & \\
\hline & Concordo totalmente & 5 & 18,5 & & & \\
\hline & Concordo parcialmente & 3 & 11,1 & & & \\
\hline Válido & Neutro & 3 & 11,1 & 0,078 & $0,015^{*}$ & 0,129 \\
\hline & Discordo parcialmente & 4 & 14,8 & & & \\
\hline & Discordo totalmente & 12 & 44,4 & & & \\
\hline
\end{tabular}

De forma geral acho fácil usar

\begin{tabular}{|c|c|c|c|c|c|c|}
\hline \multirow{5}{*}{ Válido } & Concordo totalmente & 17 & 63,0 & \multirow{5}{*}{$0,008^{*}$} & \multirow{5}{*}{0,052} & \multirow{5}{*}{0,743} \\
\hline & Concordo parcialmente & 6 & 22,2 & & & \\
\hline & Discordo parcialmente & 1 & 3,7 & & & \\
\hline & Discordo totalmente & 3 & 11,1 & & & \\
\hline & Total & 27 & 100,0 & & & \\
\hline
\end{tabular}

valor $<0,05$ Qui-quadrado de Pearson

TF= Tempo de Formação; TU = Tempo de Unidade

\section{DISCUSSÃO}

O presente estudo apresentou predominância dos participantes do gênero feminino, corroborando 
com estudos anteriores como ${ }^{14,15}$, por outro lado, discordou dos mesmos, na idade média dos profissionais, que nos seus respectivos estudos encontraram a maior frequência dos profissionais participantes no intervalo entre 20 e 30 anos. Pode-se relacionar tal fato com o tempo de formação destes profissionais, onde que segundo nosso estudo tem como predominância dos entrevistados com menos de 5 anos de conclusão de curso, enquanto outros estudos com objetivos similares encontram um tempo de formação profissional entre $6-10$ anos $^{14}$.

Sobre o tempo de atuação, encontramos resultados convergentes com Souza ${ }^{15}$ com profissionais que estão a menos de 5 anos atuando nas suas respectivas unidades. Encontramos bastante relevância no dado sobre os CD's que usam computador fora da unidade, que foi de encontro aos resultados do estudo de Silveira ${ }^{16}$, evidenciando o número considerável dos usuários dessa ferramenta.

Assim como em outras áreas do conhecimento, o uso de tecnologias de informação e comunicação na Odontologia constituem ferramentas de crescente importância ${ }^{17}$, corroborando com essa afirmação, nossos entrevistados se mostraram favoráveis ao investimento em informática no serviço de saúde e, inclusive, melhorará o serviço. Os participantes mostraram- se, ainda, satisfeitos com o sistema implantado e afirmaram que houve diferencial no serviço após a implantação, indo de encontro ao estudo de Dotta e Serra ${ }^{18}$ que afirmam que a tecnologia infere rapidez ao serviço e maior qualidade e agilidade nas ações.

Umas das etapas essenciais para a boa dinâmica do PEC é o período e tempo de treinamento, e o estudo evidenciou um número acentuado de entrevistados que considerou insuficiente a carga horária do mesmo, sendo assim incapaz de suprir as dúvidas dos profissionais, dado esse, que confronta o estudo de Souza ${ }^{15}$ que afirma que o treinamento deve conseguir suprir as dúvidas dos profissionais.

Como todas as ferramentas tecnológicas, o PEC também apresenta seus itens com menor desempenho, e como resposta ao questionamento sobre esse questionamento, encontramos a mesma de Santos et al. ${ }^{19}$ que apontou a queda de sistema como mais comum.

No tocante à percepção da facilidade do uso do PEC, Souza ${ }^{15}$ obteve como resultado um relevante número de entrevistados que sentiram- se frustrados ao operar o sistema, dado que diverge consideravelmente do nosso estudo, por outro lado, nosso estudo vai de encontro a Greiver et al. ${ }^{20}$ que observaram que alguns participantes classificaram os registros eletrônicos como complexos e inflexíveis, entretanto, atribuíram essas características às interfaces dos modelos utilizados, componentes estes que podem ser aprimorados com o desenvolvimento dos softwares. A respeito da percepção de utilidade do PEC, nosso estudo corrobora com Soranz et al. ${ }^{21}$, que afirmam que o PEC aumenta a eficiência do trabalho, enquanto se mantém em neutralidade em relação a outro estudo ${ }^{22}$ realizado no sul do Brasil, no qual é sustentado que o PEC aumenta a agilidade do trabalho.

Um ponto bem relevante do nosso estudo foi a identificação de percalços por parte dos profissionais em operar o sistema, o classificando como difícil, relatos de muito esforço para aprender a operar o PEC e que de modo geral acham o PEC difícil, relacionados respectivamente ao tempo de atuação na unidade, de formação e aumento da idade, conflitando com Gregor et al. (2002), ao citar que as interfaces humano-computador para a maior parte dos sistemas computacionais de uso geral foram desenhadas para um usuário "típico" e jovem.

CONCLUSÃO

A partir da presente pesquisa, pode-se concluir que os cirurgiões-dentistas que trabalham nas Unidades Básicas de Saúde de Patos são em sua maioria adultos jovens, com pouco tempo de formação. Pode-se inferir ainda que o Prontuário Eletrônico do Cidadão trouxe maior agilidade e eficiência ao trabalho na atenção básica, ainda existindo pontos divergentes que precisam ser superados, como o tempo de treinamento para uso da ferramenta, que acarretará uma maior aceitação e facilidade de uso.

\section{REFERÊNCIAS}

1. Morais AC. Prontuário Eletrônico do Paciente. Curitiba: Universidade Federal do Paraná, 2006.

2. Galvão MCB, Ricarte ILM. O prontuário eletrônico do paciente no século XXI: contribuições necessárias da ciência da informação. InCID Rev Ci Inf Doc. 2011; 2(2):77-100.

3. Lopes VJ, Carvalho D. KDD Na avaliação da usabilidade do prontuário eletrônico do paciente por profissionais da enfermagem. $\mathrm{R}$ Bras Inov Tecnol Saúde. 2016;6(3):21-31.

4. Muylder CF, Carneiro SD, Barros LC, Oliveira JG. Prontuário eletrônico do paciente: aceitação de tecnologia por profissionais da saúde da região metropolitana de Belo Horizonte. RAHIS. 2017; 14(1):40-52.

5. Pinto VB. Prontuário eletrônico do paciente: documento técnico de informação e comunicação do dominio da saúde . Eletr Bibliotecon Ci Inf. 2006; 2(21):34-48.

6. Saraiva AS. A importância do prontuário odontológico - com ênfase nos documentos digitais. Rev Bras Odontol. 2011;68(2):157-60.

7. Machado MJF. Prontuário Eletrônico do cidadão. Fortaleza: Encontros Universitários da UFC, v. 1, 2016.

8. Brasil. Ministério da Saúde. Resolução no 7, de 24 de novembro de 2016. 
9. Brasil. Ministério da Saúde. Departamento as Atenção Básica. Prontuário eletrônico do cidadão, 2016.

10. Fraige A. Prontuário eletrônico do centro de atendimento a pacientes especiais: desenvolvimento e implementação [tese]. São Paulo: Faculdade de Odontologia da USP; 2007.

11. Majewski CC. Pesquisa de satisfação de usuários do sistema de prontuário eletrônico do complexo hospitalar Santa Casa de Misericórdia de Porto Alegre [dissertação]. Porto Alegre: Escola de Engenharia, URGS; 2003.

12. Davis JR, Fred D. A Technology acceptance model for empirically testing new end-userinformation system: theory and results - MIT, 1986.

13. Beiguelman B. Curso prático de bioestatística -4 . ed. Ribeirão Preto: Sociedade Brasileira de Genética; 1996.

14. Lolli LF. Documentos digitais em Odontologia aspectos de legalidade, conhecimento e utilização por cirurgiões-dentistas. UNI Review. 2011; $8(2): 112-21$.

15. Souza RS. Prontuário eletrônico: ótica do profissional de saúde da atenção primária [dissertação]. Porto Alegre: Universidade Federal do Rio Grande do Sul, Faculdade de Medicina, Programa de Pós-Graduação em Ensino na Saúde; 2017.

16. Silveira LGG. Avaliação do uso da informática pelo CD e acadêmico em Odontologia. RGO. 2006;54(2):119-22.

17. Fontanella V, Schardosim M, Lara M. Tecnologias de informação e comunicação no ensino da odontologia. Rev ABENO. 2007;7(1):76-81.

18. Dotta EAV, Serra MC. Conhecimento e utilização da informática pelo cirurgião-dentista como ferramenta de trabalho. RGO (Porto Alegre). 2006;54(2):99-102.

19. Santos RPS. Análise da competência em informação mediante a transição do prontuário físico para o eletrônico. Rev Cub Inf Cienc Salud. 2017;28(4):1-13.

20. Greiver M, Barnsley J, Glazier RH, Moineddin R, Harvey BJ. Implementation of electronic medical records: effect on the provision of preventive services in a pay-for-performance environment. Can Fam Physician. 2011;57(10):e381-89.

21. Soranz D, Pinto LP, Camacho LAB. Análise dos atributos dos cuidados primários em saúde utilizando os prontuários eletrônicos na cidade do Rio de Janeiro. Ciênc saúde coletiva. 2017;22(3):819-830.

22. Silva CA, Pereira MR. Estudo e Análise das Tecnologias de Informação e Comunicação (tic) na Odontologia no Rio Grande do Sul [monografia]. Porto Alegre: Universidade Federal do Rio Grande do Sul, UFRGS; 2013.

23. Gregor P, Newell AF, Zagicek M. Designing for dynamic diversity - interfaces for older people. International ACM SIGCAPH Conference on Assistive Technologies. 2002:151-56.

\section{CONFLITO DE INTERESSES}

Os autores declaram não haver conflitos de interesse.

\section{AUTOR PARA CORRESPONDÊNCIA}

\section{Danilo Vieira Barbosa}

Universidade Federal de Campina Grande (UFCG)

danilo_vireira23@hotmail.com

Submetido em 09/09/2019

Aceito em 20/04/2020 